\title{
Case of Follicular Mucinosis Showing Brownish Yellow and Red Dots via Dermoscopy
}

\author{
Hiroki Yamagishi ${ }^{1}$, Mayumi Ota ${ }^{1}$, Yoshimasa Nobeyama ${ }^{2}$, and Akihiko Asahina ${ }^{1}$ \\ ${ }^{1}$ Jikei University School of Medicine \\ ${ }^{2}$ The Jikei University School of Medicine
}

March 2, 2022

\begin{abstract}
We reported a case of 68-year-old man suffering from follicular mucinosis. The dermoscopic examination showed multiple round brownish yellow dots with a whitish rim in the follicular ostium and red dots in the interfollicular area. This case report at first suggests that follicular mucinosis may show such dermoscopic findings.
\end{abstract}

\section{Case of Follicular Mucinosis Showing Brownish Yellow and Red Dots via Dermoscopy}

Hiroki Yamagishi, $\mathrm{MD}^{1}$, Mayumi Ota, $\mathrm{MD}^{1}$, Yoshimasa Nobeyama, $\mathrm{MD}^{1}$, and Akihiko Asahina, MD ${ }^{1}$

1. Department of Dermatology, The Jikei University School of Medicine, Tokyo, Japan

Corresponding author: Yoshimasa Nobeyama, MD, PhD

Department of Dermatology, The Jikei University School of Medicine

25-8 Nishi-Shimbashi 3-chome, Minato-ku, Tokyo 105-8461, Japan

Tel.: +81-3-3433-1111 ext. 3341; Fax: +81-3-5401-0125

E-mail: nobederm@jikei.ac.jp

The authors received no financial support for this study.

The authors have no conflicts of interest to declare.

567 words, 1 figure

Key words: dermatology

\section{ABSTRACT}

We reported a case of 68 -year-old man suffering from follicular mucinosis. The dermoscopic examination showed multiple round brownish yellow dots with a whitish rim in the follicular ostium and red dots in the interfollicular area. This case report at first suggests that follicular mucinosis may show such dermoscopic findings.

\section{KEY CLINICAL MESSAGE}

Follicular mucinosis may show the combination of both brownish yellow dots and red dots dermoscopically. Dermatologists should consider the possibility of follicular mucinosis when such dermoscopic findings are obtained.

\section{INTRODUCTION}


Follicular mucinosis, also referred to as alopecia mucinosa, was initially reported as localized alopecia histopathologically characterized by the deposition of mucin within hair follicles ${ }^{1}$. The disease is classified as idiopathic follicular mucinosis, which is not associated with other cutaneous or extracutaneous diseases, and as lymphoma-associated follicular mucinosis, which is associated with mycosis fungoides or Sézary syndrome $^{2}$. Idiopathic follicular mucinosis commonly appears as follicular papules or indurated plaques ${ }^{3}$. We herein report a case of idiopathic follicular mucinosis showing characteristic dermoscopic findings.

\section{CASE REPORT}

A 68-year-old Japanese man with a reddish plaque on the forehead for several years and alopecia on the scalp for 9 months was referred to our department. A physical examination revealed alopecic reddish plaques measuring $3 \mathrm{~cm}$ in diameter on the forehead and scalp (Figure 1a). Lymph nodes were not swollen around the head or neck. A dermoscopic examination of the reddish plaque on the forehead showed multiple round brownish yellow dots with a whitish rim in the follicular ostium and red dots in the interfollicular area (Figure 1b). A peripheral blood examination revealed no increases in the level of the soluble interleukin 2 receptor or the number of atypical lymphocytes. A histopathological examination with hematoxylin \& eosin staining showed i) an eosinophilic substance in the dilated infundibulum and a pale basophilic substance in the isthmus and outer root sheath (Figure 1c), ii) focal follicular spongiosis and vacuolar alterations at the interface between the outer root sheath and the dermis, iii) the follicular and perifollicular infiltration of lymphocytes without atypia, and iv) dilated capillary vessels in the upper dermis. The substance deposited in the hair follicle was stained with Alcian blue at pH 2.5 (Figure 1d). Based on the findings obtained, the patient was diagnosed with idiopathic follicular mucinosis and the relevant alopecia. The patient was treated 4 times each month with triamcinolone acetonide injections, with resulted in the attenuation of lesions after 4 months (Figure 1e).

\section{DISCUSSION}

Only one English study has described multiple papular lesions on an erythematous background as the dermoscopic finding of follicular mucinosis ${ }^{3}$. In the present case, the histopathological finding of mucin deposits in the follicular infundibulum and isthmus appear to correspond to the dermoscopic findings of brownish yellow dots in the follicular ostium. Also, the histopathological findings of dilated capillary vessels appear to correspond to the dermoscopic findings of red dots in the interfollicular area.

Yellow dots are a trichoscopic finding that corresponds to a dilated follicular infundibulum filled with keratotic material and/or sebum. They are observed as rounded or polycyclic structures with colors ranging from pinkish yellow to brownish yellow ${ }^{4}$. This finding has been subsequently detected in various diseases, such as alopecia areata, chronic cutaneous lupus erythematosus, androgenetic alopecia, dissecting cellulitis, and, less frequently, trichotillomania ${ }^{5}$. Therefore, brownish yellow dots in the present case are not specific to follicular mucinosis.

Red dots are a dermoscopic finding that corresponds to dilated vessels aligned perpendicular to the skin surface ${ }^{6}$. This finding has been subsequently detected in melanocytic lesions such as melanoma and melanocytic nevus as well as inflammatory diseases such as psoriasis ${ }^{6}$. Therefore, red dots in the present case are not specific to follicular mucinosis. In that context, the combination of both brownish yellow dots and red dots appears to be non-specific. However, this case report suggests that dermatologists should consider the possibility of follicular mucinosis when such dermoscopic findings are obtained.

\section{ACKNOWLEDGMENTS}

None.

\section{CONFLICT OF INTEREST}

None declared.

\section{AUTHOR CONTRIBUTIONS}


HY: contributed to validation and writing. MO: provided resources and contributed to data curation. YN: performed project administration. AA: provided supervision.

\section{ETHICAL APPROVAL}

The case report was approved by the ethics committee of The Jikei University School of Medicine and written informed consent was obtained from the patient.

\section{REFERENCES}

1 Pinkus H. Alopecia mucinosa; inflammatory plaques with alopecia characterized by root-sheath mucinosis. AMA Arch Derm 1957. 76: 419-424; discussion 424-416.

2 Cerroni L, Fink-Puches R, Back B, Kerl H. Follicular mucinosis: a critical reappraisal of clinicopathologic features and association with mycosis fungoides and Sezary syndrome. Arch Dermatol 2002. 138: 182-189.

3 Akinsanya AO, Tschen JA. Follicular Mucinosis: A Case Report. Cureus 2019. 11: e4746.

4 Lima CDS, Lemes LR, Melo DF. Yellow dots in trichoscopy: relevance, clinical significance and peculiarities. An Bras Dermatol 2017. 92: 724-726.

5 Inui S, Nakajima T, Nakagawa K, Itami S. Clinical significance of dermoscopy in alopecia areata: analysis of 300 cases. Int J Dermatol 2008. 47: 688-693.

6 Martin JM, Bella-Navarro R, Jorda E. [Vascular patterns in dermoscopy]. Actas Dermosifiliogr 2012. 103: 357-375.

\section{FIGURE LEGENDS}

\section{Figure 1}

a) Clinical findings in the first visit. Reddish plaques are evident on the forehead and scalp. The plaque on the scalp forms a hairless area. b) Dermoscopic findings of the reddish plaque on the forehead. Brownish yellow dots with a whitish rim are observed in the follicular ostium. Red dots are detected in the interfollicular area. c) Histopathological findings (hematoxylin \& eosin stain, $\times 200$ ). An eosinophilic substance in the dilated infundibulum and a pale basophilic substance in the isthmus and root sheath are evident. Focal follicular spongiosis and vacuolar alterations of the interface between the outer root sheath and dermis are accompanied by follicular and perifollicular lymphocytic infiltration. Dilated capillary vessels are observed in the upper dermis. d) Histopathological findings (Alcian blue stain, $\mathrm{pH} 2.5, \times 200$ ). The substance deposited in the hair follicle is stained with Alcian blue. e) Clinical findings at 4 months after the first visit. Alopecic lesion on the scalp becomes less prominent. 


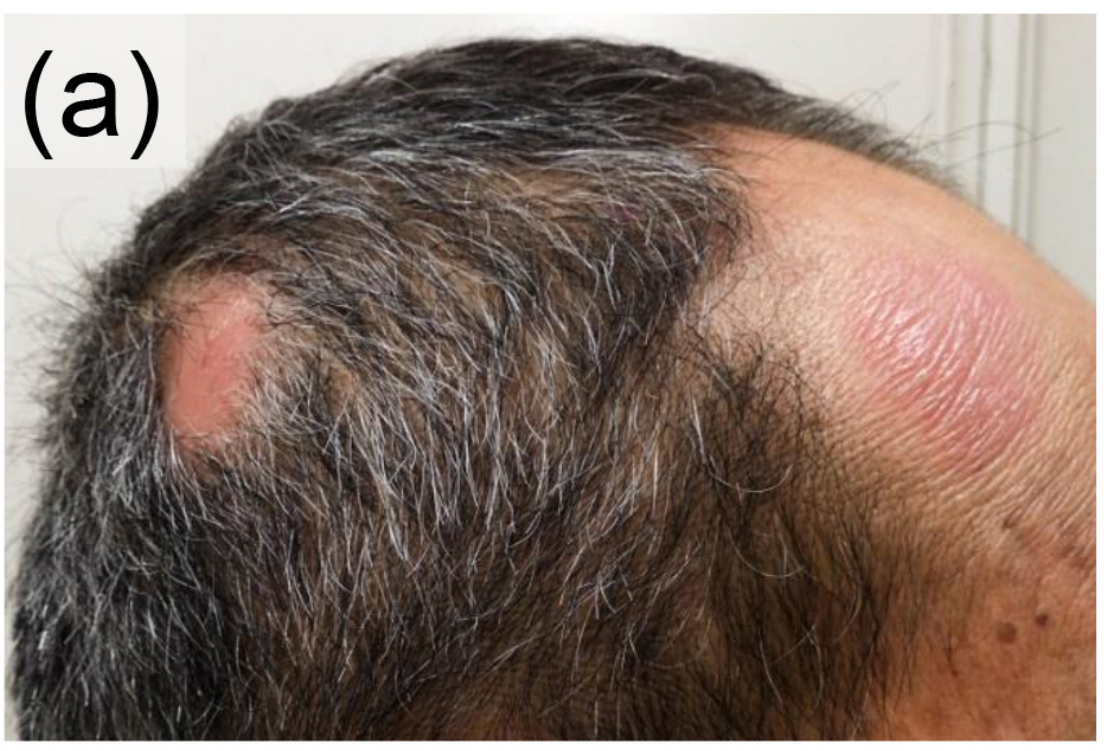

(b)
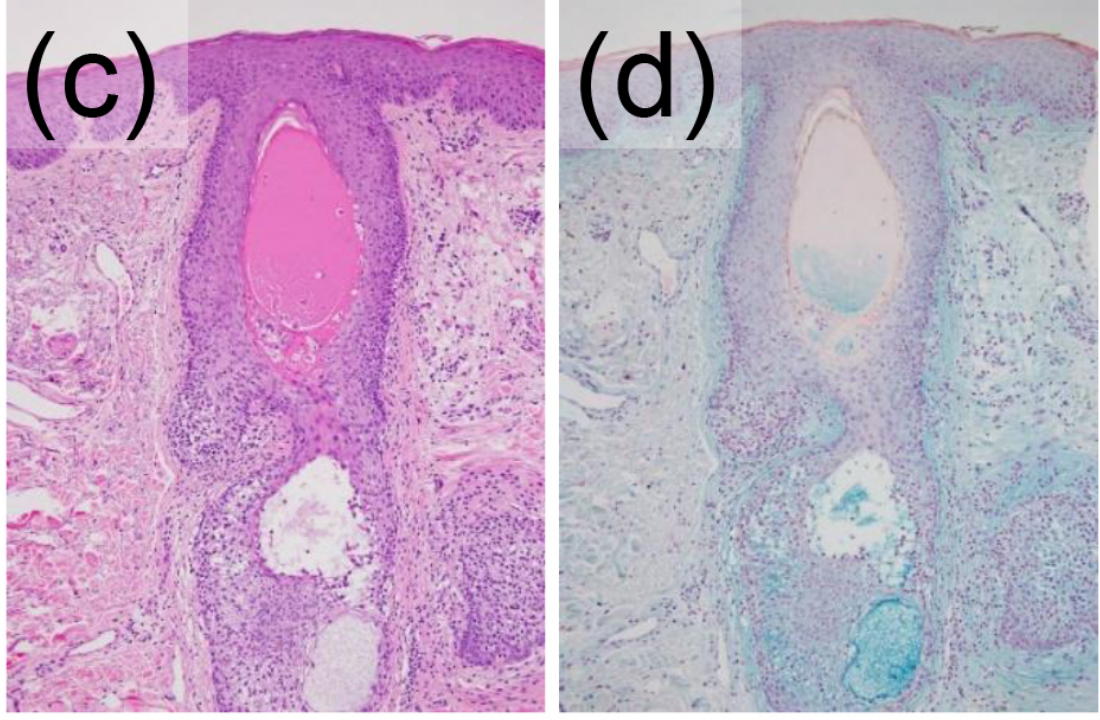

(e) 